\title{
EXISTENCE AND MULTIPLICITY OF SOLUTIONS FOR A NEUMANN PROBLEM INVOLVING VARIABLE EXPONENT GROWTH CONDITIONS
}

\author{
MARIA-MAGDALENA BOUREANU \\ Department of Mathematics, University of Craiova, 200585 Craiova, Romania \\ e-mail:mmboureanu@yahoo.com \\ and MIHAI MIHĂILESCU* \\ Department of Mathematics, Central European University, 1051 Budapest, Hungary \\ e-mail:mmihailes@yahoo.com
}

(Received 8 October 2007; revised 17 April 2008; accepted 1 May 2008)

\begin{abstract}
In this paper we study a non-linear elliptic equation involving $p(x)$ growth conditions and satisfying a Neumann boundary condition on a bounded domain. For that equation we establish the existence of two solutions using as a main tool an abstract linking argument due to Brézis and Nirenberg.
\end{abstract}

2000 Mathematics Subject Classification. 35D05, 35J60, 35J70, 58E05, 76A02.

1. Introduction. The goal of this paper is to establish the existence of solutions for the Neumann problem

$$
\begin{cases}-\operatorname{div}\left(|\nabla u|^{p(x)-2} \nabla u\right)=f(u), & \text { for } x \in \Omega \\ \frac{\partial u}{\partial v}=0, & \text { for } x \in \partial \Omega\end{cases}
$$

where $\Omega \subset \mathbb{R}^{N}(N \geq 3)$ is a bounded domain with smooth boundary, $p \in C(\bar{\Omega})$ with $1<p(x)<N$ for all $x \in \bar{\Omega}$ and $f: \mathbb{R} \rightarrow \mathbb{R}$ is a continuous function given by the formula

$$
f(t)= \begin{cases}|t|^{a-1} t, & \text { for }|t| \leq\left(\frac{1}{2}\right)^{\frac{1}{a-1}}, \\ t-|t|^{a-1} t, & \text { for }|t|>\left(\frac{1}{2}\right)^{\frac{1}{a-1}},\end{cases}
$$

where $a$ is a positive real number.

The study of problems involving variable exponent growth conditions has a strong motivation due to the fact that they can model various phenomena which arise in the study of elastic mechanics (see [27]), electrorheological fluids (see [1], [5], [14], [26]) or image restoration (see [4]). In what concern some recent studies on equations possessing variable exponent growth conditions we refer to $[\mathbf{1 0}, \mathbf{1 1}, \mathbf{1 6}-\mathbf{2 3}]$ and the references therein.

*Corresponding author. 
This paper is motivated by the studies in [17] and [18]. In [17] the following problem is studied

$$
\begin{cases}-\operatorname{div}\left(|\nabla u|^{p(x)-2} \nabla u\right)=A|u|^{a-2} u+B|u|^{b-2} u, & \text { for } x \in \Omega, \\ u=0, & \text { for } x \in \partial \Omega\end{cases}
$$

where $p \in C(\bar{\Omega})$ verifies $p(x)>1$ for any $x \in \bar{\Omega}$ and $1<a<\inf _{\Omega} p<\sup _{\Omega} p<$ $b<\min \left\{N, \frac{N \cdot \inf _{\Omega} p}{N-\inf _{\Omega} p}\right\}$ and $A, B>0$. Using Ekeland's variational principle and the mountain-pass lemma, the author shows that for $A$ and $B$ small enough problem (2) has two distinct solutions.

In [18] the following Neumann problem is analysed

$$
\begin{cases}-\operatorname{div}\left(|\nabla u|^{p(x)-2} \nabla u\right)+|u|^{p(x)-2} u=\lambda\left(|u|^{q(x)-2} u-u\right), & \text { for } x \in \Omega, \\ \frac{\partial u}{\partial v}=0, & \text { for } x \in \partial \Omega,\end{cases}
$$

where $p \in C(\bar{\Omega})$ verifies $p(x)>N$ for any $x \in \bar{\Omega}, \lambda>0$ is a constant and $q \in C(\bar{\Omega})$ satisfies $2<q(x)<\inf _{y \in \bar{\Omega}} p(y)$ for any $x \in \bar{\Omega}$. For problem (3) the author proves the existence of three solutions by using a result due to Ricceri [25].

In the present paper we continue the studies begun in [17] and [18]. Under suitable conditions we will prove the existence of two solutions for problem (1) by applying an abstract linking argument due to Brézis and Nirenberg [3]. More exactly, our key argument will be the following theorem.

TheOREM 1 (Brézis-Nirenberg [3]). Assume $X$ is a Banach space with the direct sum decomposition

$$
X=X_{1} \oplus X_{2}
$$

with $\operatorname{dim} X_{2}<\infty$. Assume $J \in C^{1}(X, \mathbb{R})$ with $J(0)=0$ satisfies $(P S)$ condition (i.e., any sequence $\left\{u_{n}\right\} \subset X$ satisfying $\left\{J\left(u_{n}\right)\right\}$ is a bounded sequence in $\mathbb{R}$ and $\left\langle J^{\prime}\left(u_{n}\right), v\right\rangle \leq \epsilon_{n}\|v\|_{X}$ for any $v \in X$, with $\epsilon_{n} \rightarrow 0$, has a convergent subsequence). Moreover, for a positive constant $R>0$, we have

$$
\begin{aligned}
& J(u) \geq 0, \text { for all } u \in X_{1} \text { with }\|u\|_{X} \leq R, \\
& J(u) \leq 0, \text { for all } u \in X_{2} \text { with }\|u\|_{X} \leq R .
\end{aligned}
$$

Also assume that $J$ is bounded below and $\inf _{X} J<0$. Then $J$ has at least two non-trivial critical points.

2. Preliminary results. In this section we recall some background facts concerning the generalized Lebesgue-Sobolev spaces $L^{p(x)}(\Omega)$ and $W^{1, p(x)}(\Omega)$, where $\Omega$ is a bounded domain in $\mathbb{R}^{N}$. We refer the reader to the book by Musielak [24] and the papers by Edmunds [6-8], Kovacik and Rákosník [15] and Fan [9, 12].

Throughout this paper we assume that $p(x)>1, p(x) \in C(\bar{\Omega})$.

Set

$$
C_{+}(\bar{\Omega})=\{h ; h \in C(\bar{\Omega}), h(x)>1 \text { for all } x \in \bar{\Omega}\} .
$$


For any $h \in C_{+}(\bar{\Omega})$ we define

$$
h^{+}=\sup _{x \in \Omega} h(x) \text { and } \quad h^{-}=\inf _{x \in \Omega} h(x) .
$$

For any $p(x) \in C_{+}(\bar{\Omega})$, we define the variable exponent Lebesgue space $L^{p(x)}(\Omega)=\left\{u ; u\right.$ is a measurable real-valued function such that $\left.\int_{\Omega}|u(x)|^{p(x)} d x<\infty\right\}$.

We define a norm, th so-called Luxemburg norm, on this space by the formula

$$
|u|_{p(x)}=\inf \left\{\mu>0 ; \int_{\Omega}\left|\frac{u(x)}{\mu}\right|^{p(x)} d x \leq 1\right\} .
$$

We remember that the variable exponent Lebesgue spaces are separable and reflexive Banach spaces. If $0<|\Omega|<\infty$ and $p_{1}, p_{2}$ are variable exponents such that $p_{1}(x) \leq$ $p_{2}(x)$ almost everywhere in $\Omega$ then there exists the continuous embedding $L^{p_{2}(x)}(\Omega) \hookrightarrow$ $L^{p_{1}(x)}(\Omega)$.

We denote by $L^{p^{\prime}(x)}(\Omega)$ the conjugate space of $L^{p(x)}(\Omega)$, where $1 / p(x)+1 / p^{\prime}(x)=1$. For any $u \in L^{p(x)}(\Omega)$ and $v \in L^{p^{\prime}(x)}(\Omega)$ the Hölder-type inequality

$$
\left|\int_{\Omega} u v d x\right| \leq\left(\frac{1}{p^{-}}+\frac{1}{p^{\prime-}}\right)|u|_{p(x)}|v|_{p^{\prime}(x)}
$$

holds true.

An important role in manipulating the generalized Lebesgue-Sobolev spaces is played by the modular of the $L^{p(x)}(\Omega)$ space, which is the mapping $\rho_{p(x)}: L^{p(x)}(\Omega) \rightarrow \mathbb{R}$ defined by

$$
\rho_{p(x)}(u)=\int_{\Omega}|u|^{p(x)} d x
$$

If $\left(u_{n}\right), u \in L^{p(x)}(\Omega)$ then the following relations hold true

$$
\begin{aligned}
|u|_{p(x)}>1 & \Rightarrow|u|_{p(x)}^{p^{-}} \leq \rho_{p(x)}(u) \leq|u|_{p(x)}^{p^{+}}, \\
|u|_{p(x)}<1 & \Rightarrow|u|_{p(x)}^{p^{+}} \leq \rho_{p(x)}(u) \leq|u|_{p(x)}^{p^{-}}, \\
\left|u_{n}-u\right|_{p(x)} \rightarrow 0 & \Leftrightarrow \quad \rho_{p(x)}\left(u_{n}-u\right) \rightarrow 0 .
\end{aligned}
$$

Next, we define

$$
W^{1, p(x)}(\Omega)=\left\{u \in L^{p(x)}(\Omega) ; \frac{\partial u}{\partial x_{i}} \in L^{p(x)}(\Omega) \text {, for any } x \in\{1, \ldots, N\}\right\} .
$$

On $W^{1, p(x)}(\Omega)$ we consider the norm

$$
\|u\|=|u|_{p(x)}+|| \nabla u||_{p(x)} .
$$

We remember that $\left(W^{1, p(x)}(\Omega),\|\cdot\|\right)$ is a reflexive and separable Banach space. 
Set

$$
\Lambda(u)=\int_{\Omega}\left(|\nabla u|^{p(x)}+|u|^{p(x)}\right) d x
$$

Then

$$
\begin{gathered}
\|u\|^{p^{-}} \leq \Lambda(u) \leq\|u\|^{p^{+}}, \quad \forall u \in W^{1, p(x)}(\Omega) \text { with }\|u\|>1, \\
\|u\|^{p^{+}} \leq \Lambda(u) \leq\|u\|^{p^{-}}, \quad \forall u \in W^{1, p(x)}(\Omega) \text { with }\|u\|<1, \\
\left\|u_{n}-u\right\| \rightarrow 0 \Leftrightarrow \Lambda\left(u_{n}-u\right) \rightarrow 0 .
\end{gathered}
$$

Finally, we note that if $s(x) \in C(\bar{\Omega})$ and $1<s(x)<p^{\star}(x)$ for all $x \in \bar{\Omega}$ then the embedding $W^{1, p(x)}(\Omega) \hookrightarrow L^{s(x)}(\Omega)$ is compact and continuous, where $p^{\star}(x)=\frac{N p(x)}{N-p(x)}$ if $p(x)<N$ or $p^{\star}(x)=+\infty$ if $p(x) \geq N$.

3. The main result. In this paper we study the existence and multiplicity of weak solutions for problem (1). We say that $u \in W^{1, p(x)}(\Omega)$ is a weak solution of (1) if

$$
\int_{\Omega}|\nabla u|^{p(x)-2} \nabla u \nabla v d x-\int_{\Omega} f(u) v d x=0,
$$

for any $v \in W^{1, p(x)}(\Omega)$.

The main result of this paper is given by the next theorem.

THEOREM 2. Assume the following inequality holds true

$$
p^{+}<a<\frac{N p^{-}}{N-p^{-}},
$$

where $a$ is given in the definition off. Then problem (1) has at least two non-trivial weak solutions.

We point out that in the context of Orlicz-Sobolev spaces a similar problem as (1) was studied recently by Halidias and Le [13]. Our result is more general than the result in [13] since the variable exponent Sobolev spaces are a special type of Musielak-Orlicz spaces which generalize the Orlicz-Sobolev spaces.

4. Proof of Theorem 2. Let $J: W^{1, p(x)}(\Omega) \rightarrow \mathbb{R}$ be the energy functional corresponding to problem (1)

$$
J(u)=\int_{\Omega} \frac{1}{p(x)}|\nabla u|^{p(x)} d x-\int_{\Omega} F(u) d x,
$$

where $F$ is a primitive of $f$, i.e.,

$$
F(t)=\int_{0}^{t} f(r) d r= \begin{cases}\frac{1}{a+1}|t|^{a+1}, & \text { for }|t| \leq\left(\frac{1}{2}\right)^{\frac{1}{a-1}} \\ \frac{t^{2}}{2}-\frac{1}{a+1}|t|^{a+1}-D, & \text { for }|t|>\left(\frac{1}{2}\right)^{\frac{1}{a-1}}\end{cases}
$$

with $D$ a positive constant such that $F$ is continuous on $\mathbb{R}$. 
Standard arguments imply that $J \in C^{1}\left(W^{1, p(x)}(\Omega), \mathbb{R}\right)$ with

$$
\left\langle J^{\prime}(u), v\right\rangle=\int_{\Omega}|\nabla u|^{p(x)-2} \nabla u \nabla v d x-\int_{\Omega} f(u) v d x,
$$

for any $u, v \in W^{1, p(x)}(\Omega)$. Thus, we observe that the critical points of functional $J$ correspond to the weak solutions of equation (1).

On the other hand, we point out that since $p^{-} \leq p(x)$ for all $x \in \bar{\Omega}$, it follows that $W^{1, p(x)}(\Omega) \subset W^{1, p^{-}}(\Omega)$.

Set

$$
V^{\prime}=\left\{u \in W^{1, p^{-}}(\Omega) ; \int_{\Omega} u(x) d x=0\right\}
$$

and

$$
V=V^{\prime} \cap W^{1, p(x)}(\Omega) .
$$

Clearly, $V^{\prime}$ is the topological complement of $\mathbb{R}$ with respect to $W^{1, p^{-}}(\Omega)$ and $V$ is the topological complement of $\mathbb{R}$ with respect to a subspace $X$ of $W^{1, p(x)}(\Omega)$, i.e.,

$$
\begin{gathered}
W^{1, p^{-}}(\Omega)=V^{\prime} \oplus \mathbb{R}, \\
X=V \oplus \mathbb{R} \subset W^{1, p(x)}(\Omega) .
\end{gathered}
$$

The above considerations show that it is enough to find weak solutions for equation (1) in the subspace $X$ of $W^{1, p(x)}(\Omega)$.

Remark 1. We remark that using the Poincaré-Wirtinger inequality (see [2], p. 194) we have

$$
|u|_{p^{-}} \leq C \cdot\|\nabla u\|_{p^{-}}, \quad \forall u \in V^{\prime}
$$

where $C>0$ is constant.

Our idea is to prove Theorem 2 by applying Theorem 1 . With that end in view, we prove some auxiliary results which show that functional $J$ satisfies the conditions from the hypotheses of Theorem 1 .

LEMMA 1. Assume that condition (11) is fulfilled. Then J is bounded from below and $\inf _{X} J<0$.

Proof. Clearly, by the definition of function $F$ we observe that $F(t) \leq 0$ for $t$ large enough. Since $F$ is continuous on $\mathbb{R}$ we deduce that there exists a constant $k>0$ such that

$$
\int_{\Omega} F(u) d x \leq k, \quad \forall u \in X
$$

Thus, we find

$$
J(u) \geq \int_{\Omega} \frac{1}{p(x)}|\nabla u|^{p(x)} d x-k \geq-k>-\infty, \quad \forall u \in X,
$$

or $J$ is bounded from below. 
On the other hand, there exists a constant $t_{1}>0$ small enough such that $\int_{\Omega} F\left(t_{1}\right) d t=\int_{\Omega} \frac{1}{a+1} t_{1}{ }^{a+1} d t=\frac{1}{a+1} t_{1}{ }^{a+1}|\Omega|>0$. Using that fact we get

$$
J\left(t_{1}\right)<0 .
$$

Since any constant function is an element of $X$ we infer that $\inf _{X} J<0$. The proof of Lemma 1 is complete.

Lemma 2. Assume that condition (11) is fulfilled. Then J satisfies the (PS) condition.

Proof. Let $\left\{u_{n}\right\} \subseteq X$ be such that

$$
\left|J\left(u_{n}\right)\right| \leq M
$$

and

$$
\left|\left\langle J^{\prime}\left(u_{n}\right), \varphi\right\rangle\right| \leq \epsilon_{n}\|\varphi\|, \quad \forall \varphi \in X
$$

where $\epsilon_{n} \rightarrow 0$.

We claim that $\left\{u_{n}\right\}$ is bounded in $X$. Arguing by contradiction and passing to a subsequence, we assume that $\left\|u_{n}\right\| \rightarrow \infty$ and $\left\|u_{n}\right\|>1$.

Set

$$
v_{n}(x):=\frac{u_{n}(x)}{\left\|u_{n}\right\|} .
$$

Since $\left\{v_{n}\right\}$ is bounded in $X$ and $X$ is a reflexive Banach space we can assume that, passing eventually to a subsequence, $v_{n}$ converges weakly to $v$ in $X$. Next, since $X$ is compactly embedded in $L^{p(x)}(\Omega)$ we infer that $v_{n}$ converges strongly to $v$ in $L^{p(x)}(\Omega)$.

By (13) we have

$$
\int_{\Omega} \frac{1}{p(x)}\left|\nabla u_{n}\right|^{p(x)} d x-\int_{\Omega} F\left(u_{n}\right) d x \leq M .
$$

On the other hand, it is obvious that

$$
t^{p(x)} \geq \rho^{p^{-}} \cdot\left(\frac{t}{\rho}\right)^{p(x)}, \quad \forall t>0, \rho>1, x \in \Omega .
$$

Choosing $t=\left|\nabla u_{n}(x)\right|$ and $\rho=\left\|u_{n}\right\|>1$ we get,

$$
\left|\frac{\left|\nabla u_{n}(x)\right|}{\left\|u_{n}\right\|}\right|^{p(x)} \cdot\left\|u_{n}\right\|^{p^{-}} \leq\left|\nabla u_{n}(x)\right|^{p(x)}, \quad \forall x \in \Omega .
$$

Using (16) we deduce that

$$
\int_{\Omega} \frac{1}{p(x)}\left|\nabla v_{n}(x)\right|^{p(x)} d x \leq \frac{1}{\left\|u_{n}\right\|^{p^{-}}} \int_{\Omega} \frac{1}{p(x)}\left|\nabla u_{n}(x)\right|^{p(x)} d x .
$$

Dividing (15) by $\left\|u_{n}\right\|^{p^{-}}>1$ and using (17) we obtain

$$
\int_{\Omega} \frac{1}{p(x)}\left|\nabla v_{n}(x)\right|^{p(x)} d x \leq \int_{\Omega} \frac{F\left(u_{n}\right)}{\left\|u_{n}\right\|^{p^{-}}} d x+\frac{M}{\left\|u_{n}\right\|^{p^{-}}}, \quad \forall n .
$$


Next, we prove that

$$
\int_{\Omega} \frac{F\left(u_{n}\right)}{\left\|u_{n}\right\|^{p^{-}}} d x \rightarrow 0 .
$$

The definition of $F$ implies that there exists a constant $M_{1}>0$ such that

$$
\frac{F(t)}{|t|^{p^{-}}} \leq 0, \quad \forall|t|>M_{1}, \text { a.e. } x \in \Omega .
$$

Hence

$$
\begin{aligned}
\int_{\Omega} \frac{F\left(u_{n}\right)}{\left\|u_{n}\right\|^{p^{-}}} d x & \leq \int_{\left\{x \in \Omega ;\left|u_{n}(x)\right| \leq M_{1}\right\}} \frac{F\left(u_{n}\right)}{\left\|u_{n}\right\|^{p^{-}}} d x+\int_{\left\{x \in \Omega ;\left|u_{n}(x)\right| \geq M_{1}\right\}} \frac{F\left(u_{n}\right)}{\left|u_{n}(x)\right|^{p^{-}}} \frac{\left|u_{n}(x)\right|^{p^{-}}}{\left\|u_{n}\right\|^{p^{-}}} d x \\
& \leq \int_{\left\{x \in \Omega ;\left|u_{n}(x)\right| \leq M_{1}\right\}} \frac{F\left(u_{n}\right)}{\left\|u_{n}\right\|^{p^{-}}} d x .
\end{aligned}
$$

The above results assure that (19) holds true.

By (18) and (19) we have

$$
\int_{\Omega} \frac{1}{p(x)}\left|\nabla v_{n}\right|^{p(x)} d x \rightarrow 0,
$$

which implies $\left\|\nabla v_{n}\right\|_{p(x)} \rightarrow 0$. Since $\|_{p(x)}$ is (weakly) inferior semi-continuous, we find

$$
0 \leq\|\nabla v\|_{p(x)} \leq \liminf _{n \rightarrow \infty}\left\|\nabla v_{n}\right\|_{p(x)}=0 .
$$

Therefore $\nabla v(x)=0$ a.e. $x \in \Omega$ which yields $v \in \mathbb{R}$. It follows that

$$
\lim _{n \rightarrow \infty}\left\|\nabla\left(v_{n}-v\right)\right\|_{p(x)}=\lim _{n \rightarrow \infty}\left\|\nabla v_{n}\right\|_{p(x)}=0 .
$$

Relation (21) and the fact that $v_{n}$ converges strongly to $v$ in $L^{p(x)}(\Omega)$ imply that actually $v_{n}$ converges strongly to $v$ in $X$. That fact combined with $\left\|v_{n}\right\|=1$ shows that $v \neq 0$ and consequently $\left|u_{n}(x)\right| \rightarrow \infty$ as $n \rightarrow \infty$ a.e. $x \in \Omega$.

Next, choosing $\varphi=u_{n}$ in (14) and taking into account that relation (13) holds true, we find

$$
\begin{aligned}
& \int_{\Omega}\left[p^{-} F\left(u_{n}(x)\right)-f\left(u_{n}(x)\right) \cdot u_{n}(x)\right] d x+\int_{\Omega}\left|\nabla u_{n}\right|^{p(x)} d x-\int_{\Omega} \frac{p^{-}}{p(x)}\left|\nabla u_{n}\right|^{p(x)} d x \\
& \quad \leq M \cdot p^{-}+\epsilon_{n} \cdot\left\|u_{n}\right\| .
\end{aligned}
$$

Dividing the above inequality by $\left\|u_{n}\right\|$ we obtain

$$
\int_{\Omega} \frac{p^{-} F\left(u_{n}(x)\right)-f\left(u_{n}(x)\right) \cdot u_{n}(x)}{\left|u_{n}(x)\right|} \cdot\left|v_{n}(x)\right| d x \leq \frac{M p^{-}+\varepsilon_{n}\left\|u_{n}\right\|}{\left\|u_{n}\right\|} .
$$

Passing to the limit in the above relation we have

$$
\liminf _{n \rightarrow \infty} \int_{\Omega} \frac{p^{-} F\left(u_{n}(x)\right)-f\left(u_{n}(x)\right) \cdot u_{n}(x)}{\left|u_{n}(x)\right|} \cdot\left|v_{n}(x)\right| d x \leq 0 .
$$


The above inequality and the Fatou Lemma imply

$$
\int_{\Omega} \liminf _{n \rightarrow \infty} \frac{p^{-} F\left(u_{n}(x)\right)-f\left(u_{n}(x)\right) \cdot u_{n}(x)}{\left|u_{n}(x)\right|} \cdot\left|v_{n}(x)\right| d x \leq 0 .
$$

On the other hand, analysing the definition of functions $f$ and $F$ we deduce

$$
\lim _{|t| \rightarrow \infty} \frac{p^{-} F(t)-f(t) t}{|t|}=\lim _{|t| \rightarrow \infty} \frac{\frac{p^{-}}{2} t^{2}-\frac{p^{-}}{a+1}|t|^{a+1}-p^{-} D-t^{2}+|t|^{a+1}}{|t|}=\infty,
$$

since by relation (11) we have $a+1>p^{-}$. It follows that there exists a constant $\alpha>0$ such that

$$
\lim _{|t| \rightarrow \infty} \frac{p^{-} F(t)-f(t) t}{|t|} \geq \alpha>0 .
$$

The above inequality, relation (22) and the fact that $\left|u_{n}(x)\right| \rightarrow \infty$ as $n \rightarrow \infty$ a.e. $x \in \Omega$ imply

$$
\int_{\Omega}|v(x)| d x \leq 0
$$

But $v \neq 0$ is a constant function as we have already noticed and that is a contradiction with the above relation. In this way we have proved that $\left\{u_{n}\right\}$ is bounded in $X$. Then there exists $u \in X$ such that $u_{n}$ converges weakly to $u$ in $X$. Since $X$ is compactly embedded in any $L^{s(x)}(\Omega)$ for any $s \in(\bar{\Omega})$ with $1<s(x)<\left(N p^{-}\right) /\left(N-p^{-}\right)$for all $x \in \bar{\Omega}$ we deduce that $u_{n}$ converges strongly to $u$ in $L^{s(x)}(\Omega)$. That information and the form of $f$ and $F$ imply that

$$
\lim _{n \rightarrow \infty} \int_{\Omega} f\left(u_{n}\right)\left(u_{n}-u\right) d x=0 .
$$

In order to prove that $u_{n}$ converges strongly to $u$ in $X$ we choose $\varphi=u_{n}-u$ in (14). This yields

$$
\begin{aligned}
& \left|\int_{\Omega}\left(\left|\nabla u_{n}\right|^{p(x)-2} \nabla u_{n}-|\nabla u|^{p(x)-2} \nabla u\right)\left(\nabla u_{n}-\nabla u\right) d x\right| \\
& \quad \leq \int_{\Omega}\left|f\left(u_{n}\right)\right|\left|u_{n}-u\right| d x+\varepsilon\left\|u_{n}-u\right\|+\left.\left|\int_{\Omega}\right| \nabla u\right|^{p(x)-2} \nabla u\left(\nabla u_{n}-\nabla u\right) d x \mid .
\end{aligned}
$$

All the above pieces of information show that

$$
\left|\int_{\Omega}\left(\left|\nabla u_{n}\right|^{p(x)-2} \nabla u_{n}-|\nabla u|^{p(x)-2} \nabla u\right)\left(\nabla u_{n}-\nabla u\right) d x\right| \rightarrow 0 .
$$

The last relation, the fact that $u_{n}$ converges strongly to $u$ in $L^{p(x)}(\Omega)$ and Theorem 3.1 in [10] imply that $u_{n}$ converges strongly to $u$ in $X$, i.e., $J$ satisfies the (PS) condition. The proof of the lemma is complete.

Lemma 3. Assume that condition (11) is fulfilled. Then there exists $\rho>0$ such that for all $u \in V$ with $\|u\| \leq \rho$ we have $J(u) \geq 0$ and $J(e) \leq 0$ for all $e \in \mathbb{R}$ with $|e| \leq \rho$. 
Proof. We choose $u \in V$ with $\|u\|=\rho$, where $\rho$ is small enough and will be specified later. The definition of $F$ and relation (11) imply that for all $\epsilon>0$ there exist $\delta>0$ and $\gamma>0$ such that

$$
F(t) \leq \varepsilon|t|^{p^{+}}, \quad \forall|t| \leq \delta, \text { a.e. } x \in \Omega
$$

and

$$
F(t) \leq \varepsilon|t|^{p^{+}}+\gamma|t|^{a+1}, \quad \forall|t| \in \mathbb{R}, \text { a.e. } x \in \Omega .
$$

Since $p^{-} \leq p(x)$ for all $x \in \bar{\Omega}$ we have that $L^{p(x)}(\Omega)$ is continuously embedded in $L^{p^{-}}(\Omega)$. Thus, there exists $k_{0}>0$ such that

$$
|u|_{p^{-}} \leq k_{0}|u|_{p(x)}, \quad u \in L^{p(x)}(\Omega) .
$$

Assuming $\|u\| \leq 1$ it follows $\|\nabla u\|_{p(x)} \leq 1$. Hence by (6) we deduce that

$$
\int_{\Omega} \frac{1}{p(x)}|\nabla u(x)|^{p(x)} d x \geq \frac{1}{p^{+}}\|\nabla u\|_{p(x)}^{p^{+}} \geq C\|\nabla u\|_{p^{-}}^{p^{+}} .
$$

Inequalities (12) and (24) imply

$$
\int_{\Omega} \frac{1}{p(x)}|\nabla u(x)|^{p(x)} d x \geq C\|u\|_{W^{1, p^{-}}(\Omega)}^{p^{+}} .
$$

Relations (23) and (11) yield

$$
\int_{\Omega} F(u) d x \leq \epsilon|u|_{p^{+}}^{p^{+}}+\gamma_{1}|u|_{a+1}^{a+1} \leq \epsilon c_{1}\|u\|_{W^{1, p^{-}}(\Omega)}^{p^{+}}+\gamma_{2}\|u\|_{W^{1, p^{-}}(\Omega)}^{a+1},
$$

where $\gamma_{1}$ and $\gamma_{2}$ are positive constants.

Choosing $\epsilon$ small enough and using relations (25) and (26) we obtain

$$
J(u) \geq C\|u\|_{W^{1, p^{-}}(\Omega)}^{p^{+}}-\gamma_{1}\|u\|_{W^{1, p^{-}}(\Omega)}^{a+1} .
$$

Relations (27) and (11) show that there exists $\theta>0$ such that

$$
J(u) \geq 0, \quad \forall u \in V \text { with }\|u\|_{W^{1, p^{-}}(\Omega)} \leq \theta .
$$

Since $V \subset X \subset W^{1, p(x)}(\Omega) \subset W^{1, p^{-}}(\Omega)$, there exists $C_{0}>0$ such that

$$
\|u\|_{W^{1, p^{-}}(\Omega)} \leq C_{0}\|u\|, \quad \forall u \in V .
$$

Taking $\rho>0$ small enough, $\|u\| \leq \rho$ implies $\|u\|_{W^{1, p^{-}}(\Omega)} \leq \theta$, for all $u \in V$ and therefore

$$
J(u) \geq 0, \quad \forall u \in V \text { with }\|u\| \leq \rho .
$$

Finally, for $t \in \mathbb{R}$ considering the constant function which belongs to $X$ we have $J(t)=-\int_{\Omega} F(t) d x$. But $F(t) \geq 0$ for $|t|$ small enough. It follows that for $t \in \mathbb{R}$ small enough we have $J(t) \leq 0$. The proof of the lemma is complete.

Proof of Theorem 2 Completed. By Lemmas 1, 2 and 3 we remark that the hypotheses of Theorem 1 are fulfilled. Thus, we conclude that problem (1) has two non-trivial weak solutions. Theorem 2 is verified. 
ACKNOWLEDGEMENTS. The authors have been supported by Grant CNCSIS PNII79/2007 'Degenerate and Singular Nonlinear Processes'.

\section{REFERENCES}

1. E. Acerbi and G. Mingione, Gradient estimates for the $p(x)$-Laplacean system, J. Reine Angew. Math. 584 (2005), 117-148.

2. H. Brézis, Analyse fonctionnelle: Théorie, méthodes et applications (Masson, Paris, 1992).

3. H. Brézis and L. Nirenberg, Remarks on finding critical points, Comm. Pure Appl. Math. 44 (8-9) (1991), 939-963.

4. Y. Chen, S. Levine and M. Rao, Variable exponent, linear growth functionals in image processing, SIAM J. Appl. Math. 66 (4) (2006), 1383-1406.

5. L. Diening, Theoretical and numerical results for electrorheological fluids, $\mathrm{Ph} . \mathrm{D}$. Thesis (University of Frieburg, Germany, 2002).

6. D. E. Edmunds, J. Lang and A. Nekvinda, On $L^{p(x)}$ norms, Proc. R. Soc. Lond. Ser. A, 455 (1999), 219-225.

7. D. E. Edmunds and J. Rákosník, Density of smooth functions in $W^{k, p(x)}(\Omega)$, Proc. $R$. Soc. Lond. Ser. A, 437 (1992), 229-236.

8. D. E. Edmunds and J. Rákosník, Sobolev embedding with variable exponent, Studia Math. 143 (2000), 267-293.

9. X. Fan, J. Shen and D. Zhao, Sobolev embedding theorems for spaces $W^{k, p(x)}(\Omega)$, J. Math. Anal. Appl. 262 (2001), 749-760.

10. X. L. Fan and Q. H. Zhang, Existence of solutions for $p(x)$-Laplacian Dirichlet problem, Nonlinear Anal. 52 (2003), 1843-1852.

11. X. Fan, Q. Zhang and D. Zhao, Eigenvalues of $p(x)$-Laplacian Dirichlet problem, J. Math. Anal. Appl. 302 (2005), 306-317.

12. X. L. Fan and D. Zhao, On the spaces $L^{p(x)}(\Omega)$ and $W^{m, p(x)}(\Omega), J$. Math. Anal. Appl. 263 (2001), 424-446.

13. N. Halidias and V. K. Le, Multiple solutions for quasilinear elliptic Neumann problems in Orlicz-Sobolev spaces, Boundary Value Prob., 2005 (3) (2005), 299-306.

14. T. C. Halsey, Electrorheological fluids, Science 258 (1992), 761-766.

15. O. Kováčik and J. Rákosník, On spaces $L^{p(x)}$ and $W^{1, p(x)}$, Czechoslovak Math. J. 41 (1991), 592-618.

16. M. Mihăilescu, Elliptic problems in variable exponent spaces, Bull. Austral. Math. Soc. 74 (2006), 197-206.

17. M. Mihăilescu, Existence and multiplicity of solutions for an elliptic equation with $p(x)$-growth conditions, Glasgow Math. J. 48 (2006), 411-418.

18. M. Mihăilescu, Existence and multiplicity of solutions for a Neumann problem involving the $p(x)$-Laplace operator, Nonlinear Anal. 67 (2007), 1419-1425.

19. M. Mihăilescu, P. Pucci and V. Rădulescu, Nonhomogeneous boundary value problems in anisotropic Sobolev spaces, C. R. Acad. Sci. Paris, Ser. I 345 (2007), 561-566.

20. M. Mihăilescu, P. Pucci and V. Rădulescu, Eigenvalue problems for anisotropic quasilinear elliptic equations with variable exponent, J. Math. Anal. Appl. 340 (2008), 687698.

21. M. Mihăilescu and V. Rădulescu, A multiplicity result for a nonlinear degenerate problem arising in the theory of electrorheological fluids, Proc. R. Soc. A: Math., Phys. Eng. Sci. 462 (2006), 2625-2641.

22. M. Mihăilescu and V. Rădulescu, On a nonhomogeneous quasilinear eigenvalue problem in Sobolev spaces with variable exponent, Proc. Amer. Math. Soc. 135 (9) (2007), 2929-2937.

23. M. Mihăilescu and V. Rădulescu, Continuous spectrum for a class of nonhomogeneous differential operators, Manuscripta Mathematica 125 (2008), 157-167.

24. J. Musielak, Orlicz spaces and modular spaces, Lecture Notes in Mathematics, Vol. 1034 (Springer, Berlin, 1983).

25. B. Ricceri, On three critical points theorem, Arch. Math. (Basel) 75 (2000), 220-226.

26. M. Ruzicka, Electrorheological fluids: Modelingn and mathematical theory (SpringerVerlag, Berlin, 2002).

27. V. Zhikov, Averaging of functionals in the calculus of variations and elasticity, Math. USSR Izv. 29 (1987), 33-66. 